\title{
Presencia de Bryopsida fértil en los niveles Westfalianos del subgrup Itararé, Cuenca de Paraná, Brasil
}

\author{
Paula G. C. Amaral ${ }^{1}$, Mary Bernardes de Oliveira ${ }^{1,2}$, Fresia Ricardi- \\ Branco ${ }^{3}$, Jean Broutin ${ }^{4}$
}

1. Pós-graduação Instituto de Geociências - USP, São Paulo, SP.

2. Laboratório de Geociências - UnG, Guarulhos, SP \& Instituto de Geociências USP, São Paulo, SP. E-mail maryeliz@usp.br

3. Instituto de Geociências - UNICAMP, Campinas, SP . E-mail: fresia @ige.unicamp.br

4. Lab. de Paleobotanique et Paleoecologie, Université Pierre et Marie Curie, Paris, France

\begin{abstract}
The bryophyte fossils are rare, mainly in Paleozoic sedimentary rocks in spite of being present since the Silurian Period. In the Division Bryophyta, the fossils that belong to the Class Bryopsida are recognized since the Carboniferous, but they are extremely scarce. They are plentiful only in Permian sediments, in the Petchora, Kuznetsk and Russian Platform basins, also in Antarctica, Karoo basin (the last in South Africa) and India. Identified at the genus Dwykea, gametophyte specimens bearing pleurocarpous sporophyte were recovered from the lowermost levels of Itararé Subgroup, near Campinas city, S. Paulo State. These fossils correspond to the first register of bryophyte female gametophyte for the Carboniferous Period. The microflora in association with these fossils allow correlations of these levels to the Palynozone Ahrensisporites cristatus of Westphalian age. Related to proglacial sediments, they may correspond to a tundra vegetation covering the Northeastern border of Paraná Basin, during the Westphalian
\end{abstract}

Resumén: Los fósiles atribuidos a briófitos son escasos, principalmente en rocas paleozoicas a pesar de ser registrados desde el Silúrico. Para la División Bryophyta, los fósiles correspondientes a la Clase Bryopsida comienzan a ser encontrados a partir del Carbonífero, aunque son bastante escasos. Registros más abundantes son conocidos para el Pérmico en rocas de las cuencas de Petchora, Kuznetsk y en la Plataforma Rusa, así como en la Antártica, en la Cuenca del Karoo (África del Sur) e India. Especímenes de gametófitos con esporofitos pleurocárpicos del género Dwykea fueron colectados en los niveles inferiores del Subgrupo Itararé, próximos a la ciudad de Campinas, Estado de S. Paulo. Estos fósiles corresponden al primer registro para el periodo Carbonífero de gametofitos femeninos fértiles. La microflora asociada a los fósiles de Dwykea permite establecer correlaciones con la Palinozona Ahrensisporites cristatus de edad westfaliana. Además los niveles donde fueron colectados los ejemplares de Dwykea corresponden a sedimentos proglaciales, que son interpretados como una vegetación de tundra que habitaba en el margen noreste de la Cuenca del Paraná, durante el Westfaliano. 


\section{INTRODUCCIÓN:}

El objetivo principal de este trabajo fue estudiar impresiones de briófitos colectados en capas del Carbonífero Superior (Westafaliano), asociadas a rocas depositadas en ambientes proglaciales de la Gondwana Occidental. En las impresiones estudiadas aparece el primer esporofito pleurocárpico conocido en el registro fósil de la Clase Bryopsida, hecho que puede contribuir con el conocimiento de la historia evolutiva de los briófitos.

Los briófitos son plantas de tamaño pequeño y sin verdaderos tejidos vasculares, con estructura anatómica y textura frágil. Estas características hacen que su fosilización sea bastante difícil como aleatoria, en contraste con lo que puede ser observado en las plantas vasculares (traqueófitas) que poseen tejitos mas resistentes al transporte, deposición y a los procesos de compactación de los sedimentos. Por estas razones la mayoría de las veces en que son encontrados fósiles de briófitos, estos están fosilizados como impresiones, que pueden estar, en algunos casos, cubiertas por una fina película carbonosa, que permite la confección de estudios de la microestructura como en el caso de Ignatov (1990). Así mientras mas fino sea el sedimento y menor el transporte sufrido por la planta mejor serán en riqueza de detalles los fósiles. En condiciones especiales, como las descritas por Frahm (2003) puede ocurrir la preservación en ámbar o las plantas ser permineralizadas (Smooth \& Taylor, 1995), en ambos casos ocurre una preservación excepcional. De esta forma por lo general es extremadamente delicada en Paleontología la identificación taxonómica de los briófitos a nivel inferior que el de Clase, pues ésta es realizada con base en los caracteres morfoanatómicos de las hojas, que difícilmente fosilizan.

Los fósiles vegetales atribuidos a briófitos son muy raros, en particular para la Era Paleozoica (Taylor \& Taylor, 1993, Stewart \& Rothwell, 2001; Archangelsky \& Ottone, 2001, Frahm, 2003). Los fósiles más antiguos, encontrados hasta el presente, se remontan al Silúrico, representados por esporas con afinidades, posiblemente, relacionadas a briófitos (Taylor \& Taylor, 1993). Algunos ejemplares de gametófitos de hepáticas, incluidos dentro del género Pallavicinites (Taylor \& Taylor, 1993, Stewart \& Rothwell, 2001; Frahm, 2003) fueron encontrados en rocas del Devónico de Nueva York. (Figura 1). Solamente a partir del Carbonífero se pueden identificar fósiles de briófitos sin lugar a dudas (Jovet-Ast, 1967; Oostendorp, 1987).

Los fósiles más antiguos conocidos para la Clase Bryopsida fueron descritos a partir de ejemplares del Carbonífero Inferior (Viseano) de Gran Bretaña y clasificados en la especie Muscites plumatus, siendo relacionados con la familia actual de briófitos Dicranaceae (Cleal \&Thomas, 1995). Un siglo antes Renault \& Zeiller (1885) describieron fósiles de Bryopsida de edad estefaniana encontrados en el Macizo Central francés, como Muscites polytrichaceus. Para la Argentina, Ottone \& Archangelsky (2001) estudiaron y describieron fósiles de Muscites amplexifolius que ocurren en las formaciones Guandacol y Tupe con una edad carbonífera en la Provincia de San Juan. Todas estas especies del género Muscites describen a formas estériles, sin que sea conocido si estos primeros briófitos eran acrocárpicos o pleurocárpicos (Fahm, 2003). Aunque los registros de briófitos carboníferos son aun poco expresivos, numerosas especies con excelente preservación, han sido descritas para el Pérmico. Para Angara fueron descritos 20 géneros de briófitos, así pueden ser mencionados los trabajos de Neuburg $(1955,1960)$ para las cuencas de Petchora, Tunguska y Kuznetsk y de Ignatov (1990), para la plataforma rusa entre otros. Para el paleocontinente de Gondwana son conocidas las formas descritas por Smoot \& Taylor (1986) para fósiles permineralizados encontrados en la Antártica clasificados como Merceria augustica y posiblemente relacionados con Hypnales o Leucodontales. Anderson \& Anderson (1985), para la Cuenca de Karoo en África del Sur, pasaran para sinonimia los ejemplares de musgos citados en Plumstead (1966) y los ejemplares de cf. Dicranum citados en Lacey et al. (1975), creando el género Dwykea encontrado en rocas sedimentarias de la Formación Dwykea (Pérmico Inferior). Otro género de briófitos gondwanicos de la Cuenca del Karro es Buthelezia (Lacey et al., 1975) descrito para el Permico Superior. Para la India 


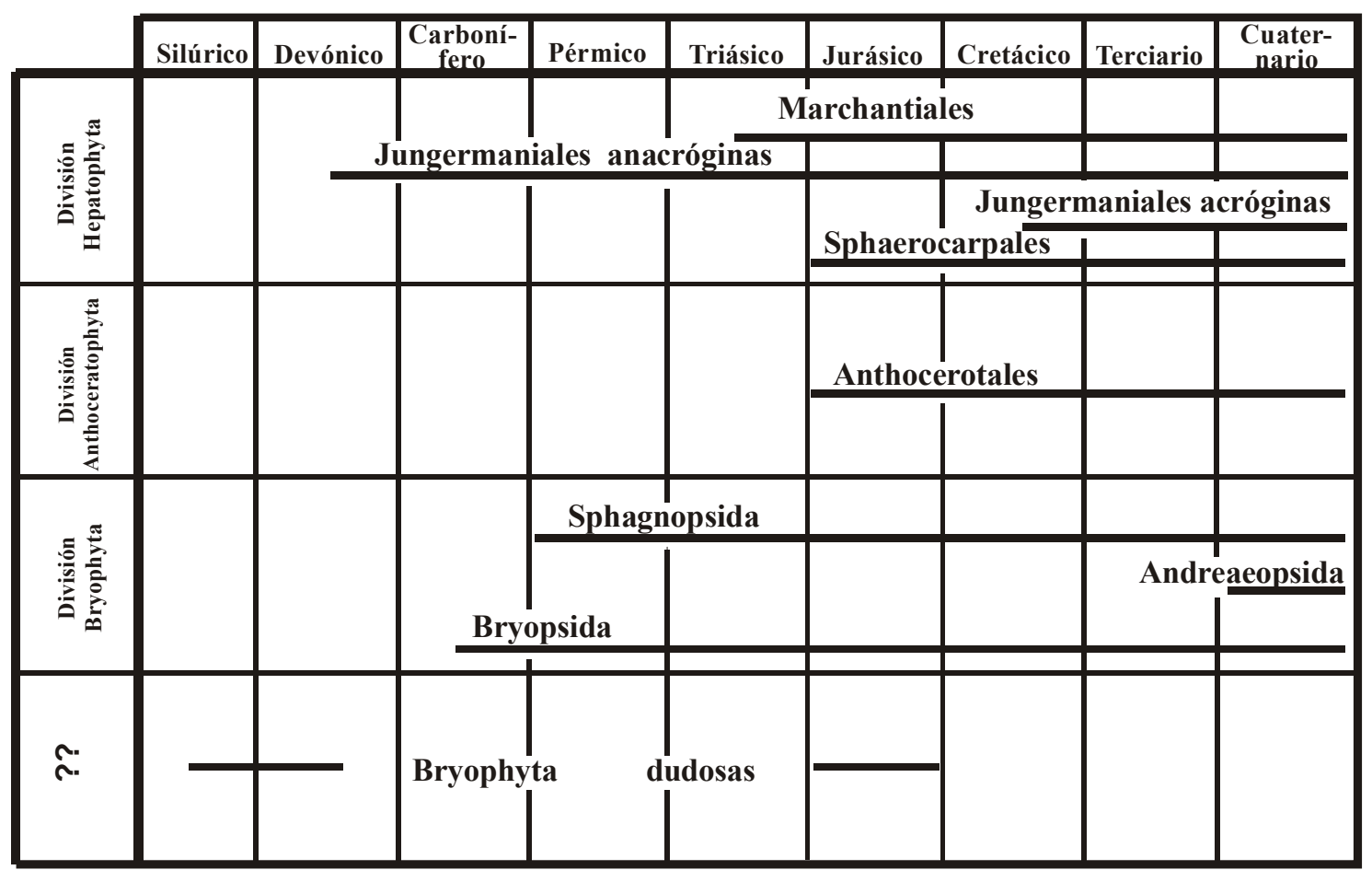

Figura 1: Distribución estratigráfica de las divisiones de Bryophyta. (modificado de Jovet-Ast, 1967: 173 Tab. II; Taylor \& Taylor, 1993; Stewart \& Rothwell, 2001; Frahm, 2003).

Chandra (1996) describió varios géneros como Saksenaphyllites, Talchirophyllites y Umariaphyllites. El registro fosilífero de briófitos conocido para las eras mesozoica y cenozoica, no será aquí discutido por estar fuera del propósito del presente trabajo.

\section{Localización geográfica y Geología:}

El sitio donde fueron colectados los fósiles está geográficamente localizado en el Km. 96 de la Autopista de Los Bandeirantes, que une a las ciudades de S. Paulo y Campinas (Figura 2). En esta localidad se encuentran expuestas rocas sedimentarias del Carbonífero Superior (Westfaliano). El afloramiento en si, posee una extensión aproximada de $100 \mathrm{~m}$ por $20 \mathrm{~m}$ de altura. En él fue observada la presencia de más de 50 capas de limonitas de color gris claro y pertenece al Subgrupo Itararé (Amaral, 2000). Esta secuencia proglacial de rocas sedimentarias fué depositada cercana a la margen de un mar por acción del deshielo de lenguas glaciales, relacionado con el final de la glaciación ocurrida en la porción sur del Gondwana durante el Carbonífero. En el mismo afloramiento fueron también encontrados abundantes megásporas y palinomorfos (granos de polen y esporas, Amaral, 2000). Al correlacionar los palinomorfos encontrados con la bioestratigrafía vigente de la porción noreste de la Cuenca del Paraná fué encontrada la edad del afloramiento, el que pertenece a la Palinozona Ahrensisporites cristatus de edad westfaliana. (Souza, 2000). Así la paleoflora encontrada en el afloramiento del Km 96 de la Autopista de Los Bandeirantes es compuesta por megásporas de licófitas y esfenófitas, además de caules no identificados y briófitos.

Los ejemplares estudiados están fosilizados como impresiones y forman parte de la Colección Científica de Tipos (3T) del Laboratorio de Sistemática Paleontológica del Departamento de Geología Sedimentar e Ambiental, Instituto de Geociências, 


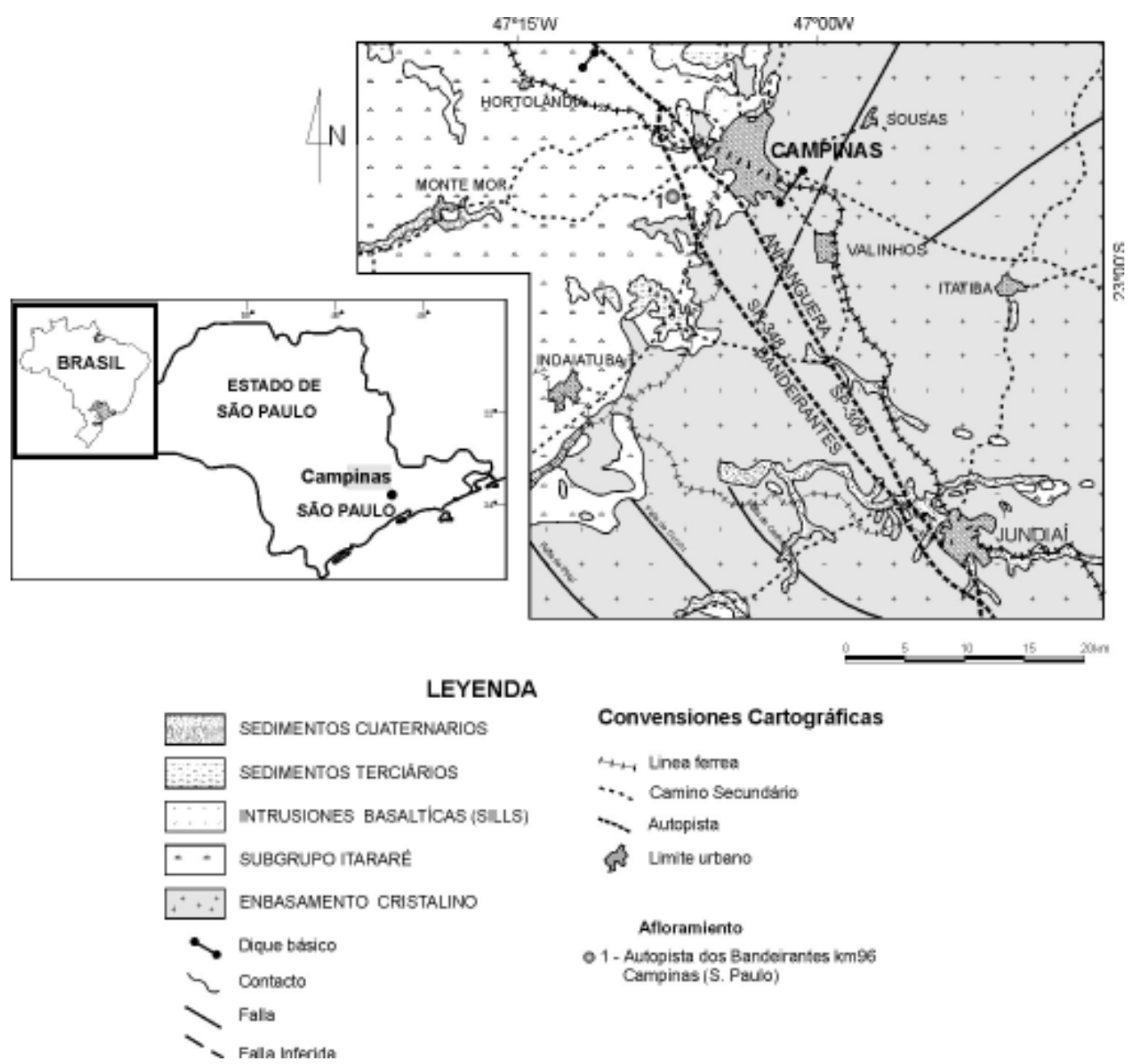

Figura 2: Mapa geológico de la región de Campinas, Estado de S. Paulo (SP), Brasil. El número uno (1) indica la localización del afloramiento (Autopista SP - 348 Bandeirantes) local donde fueron colectados los briofitos estudiados.

Universidade de S. Paulo, Brasil, y comprenden las muestras GP/3T $2284 \mathrm{~A}$ e B.

\section{SISTEMÁTICA:}

\section{BRYOPHYTA}

Bryopsida

aff. Dwykea sp. Anderson et Anderson 1985

Planchas 1 y 2

Ejemplares GP/3T 2284 A y B
Sitio: afloramiento del Km. 96 de la Autopista de Los Bandeirantes (Campinas, Estado de São Paulo - Brasil). Figura 2.

Posición estratigráfica: porción inferior del Subgrupo Itararé (Carbonífero Superior Pérmico Inferior). Palinozona de Biointervalo Ahrensisporites cristatus.

\section{Descripción:}

Dos impresiones, parcialmente carbonizadas, se encuentran preservadas en la misma roca. Una de ellas (GP/3T 2284 A-Plancha 1) corresponde 
a un fragmento medio-apical de un gametofito femenino de briófito. El eje caulinar de $0,6 \mathrm{~mm}$ de ancho, recorrido por 2 o 3 estrías longitudinales, con longitud de 17,0 $\mathrm{mm}$. El caule porta diminutas hojas lineares y falcadas de 5,2 $\mathrm{mm}$ de largo por 0,2 $\mathrm{mm}$ de ancho máximo. La filotaxia es claramente helicoidal, las hojas tienen ápice agudo y base decurrente al eje. Costa muy fina presente que recorre longitudinalmente a las hojas.

La otra impresión GP/3T 2284B, con las mismas características morfológicas generales, presenta además un esporofito inmaduro conectado orgánicamente a la axila de una de las hojas laterales del gametofito (Plancha 2 .Figs. 1a y 1b). El esporofito pleurocárpico joven está constituido por una seta de $0,6 \mathrm{~mm}$ de longitud por $0,04 \mathrm{~mm}$ de ancho, portando una cápsula de $0,2 \mathrm{~mm}$ de longitud por $0,08 \mathrm{~mm}$ de ancho.

\section{Discusión y comentarios:}

En las impresiones estudiadas la mayoría de las hojas se encuentran parcialmente conservadas, aunque el ápice no pueda ser observado en todos ellos (Planchas 1 y 2). Con base en el gametofito folioso de simetría radial (filotaxia espiralada), en el cual es observada la costa representada por un fino filamento carbonoso que recorre las hojas y el caule, se puede incluir a las muestras estudiadas dentro de la Clase Bryopsida (musgos). Adicionalmente, en las impresiones estudiadas se encuentran representados otros caracteres propios de esta Clase como gametofito formado por un eje caulinar erecto, filotaxia helicoidal, longitud entre $0,5 \mathrm{~mm}$ y $50 \mathrm{~mm}$ y esporofito constituido por seta que presenta una cápsula.

Thomas (1972) describió un pequeño eje folioso como Muscites plumatus colectado en el arenito Drybrook de edad viseana (Pedrera Puddlkebrook), en Gran Bretaña, considerado como el fósil más antiguo de Bryopsida conocido hasta el presente. Otro fósil de Bryopsida del Carbonífero es Muscites amplexifolius (Ottone \& Archangelsky,2001) colectado en las formaciones Guandacol (intervalo Namuriano Superior - Westfafiano Inferior) y Tupe (Westfaliano - Estefaniano). Anteriormente Muscites polytechniciens (Renault \& Zeiller, 1885), había sido descrito para el Estefaniano del Macizo Central francés, caracterizado por hojas lanceolados similares al los del género actual Mnium. Por lo general, las hojas y las bases de estas formas son muchos más amplias que las de los ejemplares aquí descritos caracterizados por hojas lineares falcadas, por lo que no pueden ser incluidos en Muscites. Merceria, género de briófitos descrito para el Pérmico de la Antártica (Smoot \& Taylor, 1986) posee como características eje delicado, aunque sus hojas tienen el doble del tamaño que las muestra brasileñas y costas más gruesas, al contrario de las estudiadas. Además Merceria fué descrito para ejemplares permineralizados, lo que hace imposible su comparación con formas fosilizadas como impresiones, por presentar caracteres no fosilizados en las impresiones.

Los especimenes brasileños tienen características en común con el género Dwykea Anderson et Anderson, 1985 (Cuenca de Karoo, África del Sur ) como puede ser apreciado en la Figura 3, en lo que respecta a las dimensiones del caule, en la forma linear y de ápice agudo de las hojas y por presentar hojas muy próximas. Las diferencias observadas entre Dwykea y los ejemplares estudiados son la presencia de una costa tenue en los ejemplares estudiados no observada, hasta ahora, en Dwykea, bien como que este genero fue introducido para ejemplares estériles, por esas razones los ejemplares estudiados fueron clasificados como aff. Dwykea.

Es importante comentar que el Subgrupo Itararé (Cuenca del Paraná, Brasil) y la Formación Dwyka (Pérmico Inferior de la Cuenca del Karoo, África del Sur) son aproximadamente correlacionables una vez que, ambos fueron depositados bajo la influencia de la glaciación del Gondwana y son compuestos por rocas sedimentarias depositadas en ambientes glaciales, proglaciales, periglaciales y marinos. Las dos cuencas mencionadas (Paraná y Karoo) se encontraban muy cercanas y formaban parte de un gran mar interior localizado en la porción sureste del Gondwana occidental (Figura 4) durante gran parte de la Era Paleozoica. Estas evidencias paleogeográficas son una prueba más acerca de la relación entre los especimenes de briótifos estudiados y el género Dwykea. Aun resta saber si los ejemplares de aff. Dwykea 


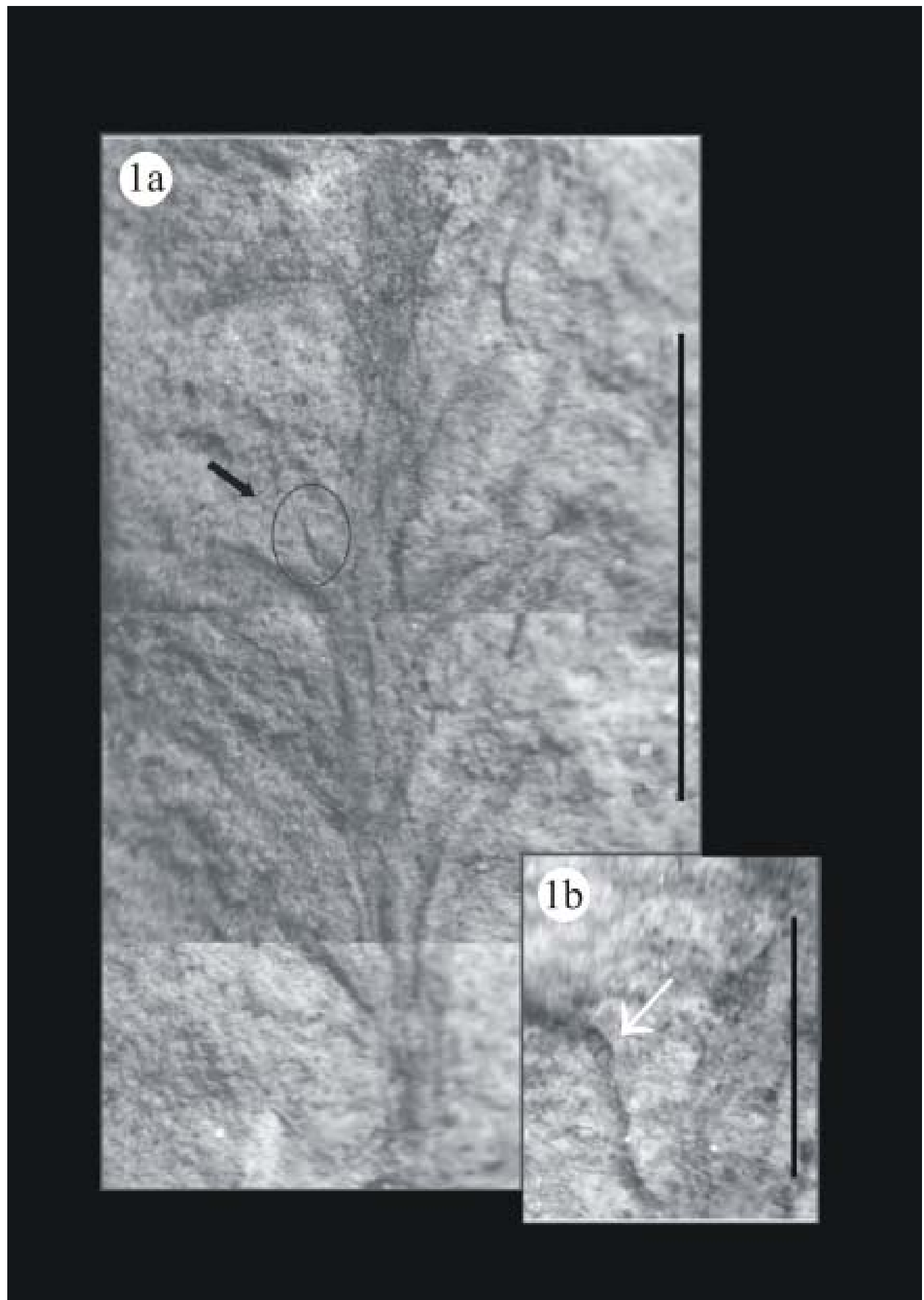

Plancha 1

TROPICAL BRYOLOGY 25 (2004) 


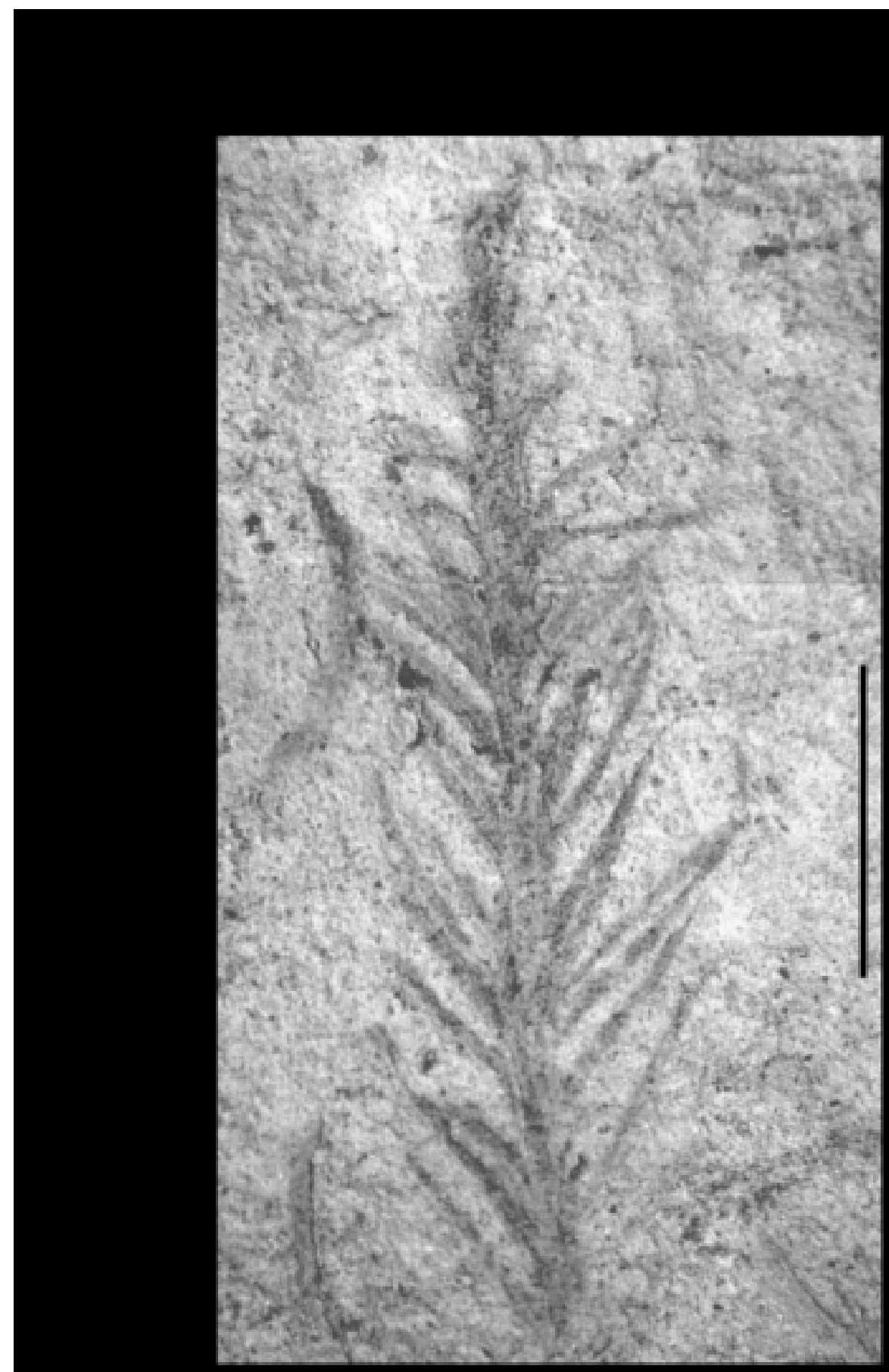

Plancha 2 


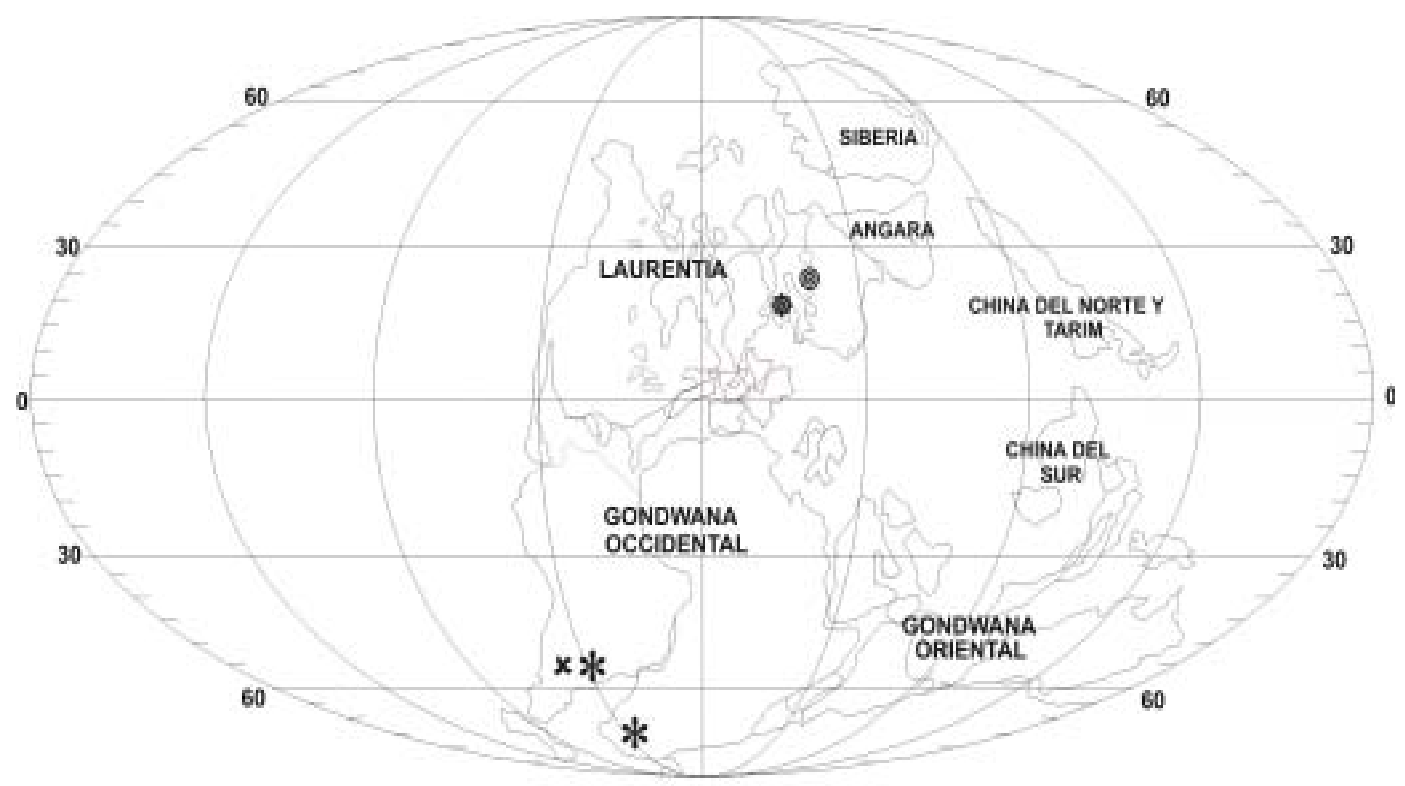

Figura 4: Paleogeografía del Carbonífero Superior.

* Ejemplares del género Dwykea . × Localización de la especie Muscites amplexifolius

Localización de las especies M. plumatus y M. polytechniciens

pertenecen a una nueva especie del género, por presentar más caracteres diagnósticos, como esporofito y costa, que los descritos en la diagnosis de Dwykea por Anderson \& Anderson (1985), estas preguntas podrán ser aclaradas en un futuro estudio, en el que se puedan estudiar las formas brasileras y las surafricanas.

\section{CONCLUSIONES:}

Por último la presencia de briófitos, más precisamente de Bryopsida, en los niveles westfalianos del Subgrupo Itararé, corresponde al registro más antiguo de formas fértiles de todo el paleocontinente de Gondwana. Una vez que Dwykea goedehoopensis fue colectada en niveles considerados del Pérmico Inferior. En conclusión, el descubrimiento de un espécimen fértil es una prueba irrefutable de la existencia de briófitos durante el Carbonífero del Gondwana. Adicionando a este hecho, las pruebas sedimentológicas (como presencia de glaciares) y palinológicas (granos de polen y esporas), podemos afirmar que los briófitos crecían en un ambiente próximo a los glaciares semejante a los que hoy son encontrados en el margen de los glaciares antárticos o en la tundra.

\section{AGRADECIMIENTOS:}

Los autores quieren expresar su mayor agradecimiento a la Fundação de Amparo à Pesquisa no Estado de São Paulo por haber subvencionado este trabajo Proyecto Temático FAPESP 97/03639-8 - Thematic Project: "Paleofloristic composition and succession survey of the Upper Carboniferous - Lower Permian (Tubarão Group) in the State of São Paulo". También agradecen al Prof. Dr. Paulo Roberto dos Santos y al Ms. Fabio Branco por su colaboración durante las colectas y por sus estimulantes discusiones, así como al referee por sus comentarios que enriquecieron mucho el presente trabajo. 


\begin{tabular}{|c|c|c|c|}
\hline & & Anderson \& Anderson (1985) & $\begin{array}{c}\text { Este } \\
\text { trabajo }\end{array}$ \\
\hline \multirow{8}{*}{ 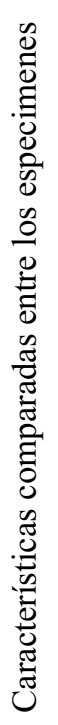 } & Longitud del eje & $>25 \mathrm{~mm}$ & $>17 \mathrm{~mm}$ \\
\hline & $\begin{array}{c}\text { Dimensiones de las hojas } \\
\text { (largo x ancho) }\end{array}$ & $5,0 \mathrm{~mm} \times 0,15 \mathrm{~mm}$ & $5,2 \mathrm{~mm} \times 0,2 \mathrm{~mm}$ \\
\hline & Distancia entre las hojas & Muy próximas & Muy próximas \\
\hline & Ápice de las hojas & Agudo & Agudo \\
\hline & Filotaxia & Poco clara & Helicoidal \\
\hline & Costa & Ausente & Tenue \\
\hline & Gametofito & No observado & Presente \\
\hline & Esporofito & No observado & Pleurocárpico \\
\hline
\end{tabular}

Figura 3: Semejanzas y diferencias entre Dwykea y los especimenes descritos. (modificado de Anderson \& Anderson, 1985 y Amaral, 2000)

\section{BIBLIOGRAFÍA:}

Amaral, P.G. C. 2000. Estudo da tafoflora do Subgrupo Itararé, no afloramento Km 96 da Rodovia dos Bandeirantes, Municipio de Campinas, SP. 39p. (Monografía de Trabajo de Grado. Instituto de Geociências, Universidade de S. Paulo, Brasil)

Anderson, J. M. \& Anderson, H.M. 1985. Paleoflora of Southern Africa Prodromus of South African megafloras Devonian to lower Cretaceous. Ed. A. A. Balkema, Pretoria, 423p.

Chandra, S. 1996. Bryophytic remains from the Early Permian sediments of India. The Palaeobotanist, 43: $16-48$.

Cleal, C.J. \& Thomas, B.A. 1995. Paleobotany of Great Britain. Ed. Chapman \& Hall, Great Britain. 460p.
Frahm, J.-P. 2003. Manual of tropical bryology. Tropical Bryology, 23: $1-28$

Ignatov, M.S. 1990. Upper Permian mosses from the Russian Plataform. Paleontographica 217B: 147189

Jovet-Ast S. 1967. Bryophyta. In Traité de paléobotanique, II, Bryophyta, Psilophyta, Lycophyta (E. Boureau, Ed.), Masson et Cie, Paris : $17-186$

Lacey, W S., Dijk, D.E. van \& Gordon-Gray, K D. 1975. Fossil plants from the Mooi River district of Natal, South Africa. Annals of the Natal Museum, 22: $81-109$.

Neuburg, M.F. 1955. New representative of the Lower Permian flora of Angara. Akad. Nauk., S.S.S.R. Doklady, Repts. Paleont., 102: 613 616 
Neuburg, M.F. 1960. Leafy mosses from the Permian deposits of Angaraland. Trudy Geol. Inst. Akad. Nauk, 19: 1 - 104

Oostendorp, C. 1987. The Bryophytes of the Paleozoic and the Mesozoic (Bryophytorum Bibliotheca, 34) J. Cramer, Berlin : 112 p.

Ottone, E.G. \& Archangelsky, S. 2001. A new bryophyte from the Upper Carboniferous of Argentina. Amaghiniana, 32: 219 - 223.

Plumstead, E. 1966. Recent palaeobotanical advances and problems in Africa. In: Symposium on floristic and stratigraphy of Gondwanaland, Luknow. pp. 1 - 12.

Renault, B. \& Zeiller, R. 1885. Sur des mousses de la epoque houillére. Compt. Rendu. Acad. Sci. 100: $660-662$.

Smoot, E. \& Taylor, T. N. 1986. Structurally preserve fossil plants from Antarctica: II. a Permian moss from the Antarctic Mountains. Amer. J. Bot. 73: $1683-1691$.

Souza, PA. 2000. Palinobioestratigrafia do Subgrupo Itararé, Carbonífero/Permiano, na porção nordeste da bacia do Paraná (SP/PR, Brasil). 299p, inédito (Tese de Doutoramento - Instituto de Geociências da USP)

Stewart, W. N. \& Rothwell, G. W. 2001. Paleobotany and the evolution of plants. 2 ed. Cambridge University Press. Edinburg. 521p.

Taylor, T. N. \& Taylor, E. L. 1993. The Biology and evolution of fossils plants. Prentice Hall. New Jersey. 982p.

Thomas, B.A. 1972. A probable moss from the Lower Carboniferous of the Forest of Dean, Gloucestershire. Annals of Botany 36: 155 - 161. 